\begin{tabular}{c|c|c|c}
\hline Condizione di Partenza & Condizione Accessoria A & Condizione Accessoria B & Probabilità di Decesso \\
\hline Positivo al COVID-19 & $/$ & $/$ & $5 \mathrm{su} 1.000$ \\
Positivo al COVID-19 & Età Inferiore ai 60 Anni & $/$ & $25 \mathrm{su} 10.000$ \\
Positivo al COVID-19 & $/$ & Sano PRIMA del Contagio & $18 \mathrm{su} 10.000$ \\
Positivo al COVID-19 & Età Inferiore ai 60 Anni & Sano PRIMA del Contagio & 9 su 100.000 \\
NON Positivo al COVID-19 & Età Inferiore ai 60 Anni & Sano PRIMA del Contagio & da 9 a 80 su 1 Milione
\end{tabular}

\title{
COVID-19 in Italia: una Percezione Amplificata del Rischio? [COVID-19 in Italy: an amplified risk perception?]
}

\author{
Authors: $\quad$ Carmine Cataldo \\ Submitted: $\quad$ 29. April 2020 \\ Published: $\quad$ 8. May 2020 \\ Volume: 7 \\ Issue: $\quad 3$ \\ Affiliation: Independent Researcher, $\mathrm{PhD}$ in Mechanical Engineering, MD in \\ Disciplines of Jazz and Improvisation (Jazz Piano), Battipaglia \\ (SA), Italy \\ Languages: Italian \\ Keywords: $\quad$ COVID-19, Italia, Tasso di Letalità, Comorbilità, Lockdown, \\ Lombardia [COVID-19, Italy, Case Fatality Rate, Comorbidities, \\ Lockdown, Lombardy.] \\ Categories: Medicine \\ DOI: $\quad$ 10.17160/josha.7.3.670
}

Abstract:

In Italy, the perception of the risks related to COVID-19 may be considerably amplified. In this brief report we initially propose a realistic value for the Case Fatality Rate of COVID-19, obtained by taking into account hidden deaths and infections. Subsequently, the CFR is modified by considering age and comorbidities. In order to concretely weigh up the risks related to COVID-19, we also derive the CFR for smoking, road and domestic accident. A short section is dedicated to the Lombardy anomalous scenario.

\section{JOSHA

Journal of Science,

Humanities and Arts 


\title{
COVID-19 in Italia: una Percezione Amplificata del Rischio?
}

\section{By Carmine Cataldo}

$\mathrm{PhD}$ in Mechanical Engineering, Battipaglia (SA), Italy

\begin{abstract}
II presente report si pone l'obiettivo di far luce sulla controversa stima del Tasso di Letalità del COVID-19. Nella prima sezione, il Tasso di Letalità è calcolato dapprima in forma grezza, avvalendosi dei dati resi disponibili dalla Protezione Civile, e successivamente in forma corretta, impiegando coefficienti di correzione derivati riferendosi a stime largamente riconosciute come affidabili. Nella seconda sezione, al fine di fornire al lettore alcuni termini di paragone efficaci, vengono calcolati i Tassi di Letalità relativi al tabagismo, ai sinistri stradali, agli incidenti domestici. Inoltre, per completezza di trattazione, vengono riportati $\mathrm{i}$ Tassi di Letalità per alcune ben note patologie. Nella terza sezione viene affrontata la caratterizzazione dei decessi. La procedura consente di pervenire alla stima delle probabilità di decesso per un individuo sano, d'età inferiore ai 60 anni, non ancora positivo al virus. Nella quarta e ultima sezione viene brevemente discussa l'anomalia lombarda. II documento è stato redatto avendo cura di renderne il contenuto comprensibile al maggior numero di utenti. Gli elementi di calcolo delle probabilità cui si è fatto ricorso sono meno che elementari. II lettore può verificare la veridicità dei dati riportati, numerici e non, consultando le fonti espressamente citate.
\end{abstract}

Keywords

COVID-19, Italia, Tasso di Letalità, Comorbilità, Età Pazienti, Probabilità Decessi, Lombardia, Lockdown. 


\section{COVID-19: Tasso di Letalità}

\subsection{Tasso di Letalità Grezzo}

II Tasso di Letalità è fornito dal rapporto tra il numero dei decessi e quello dei contagi. II Tasso di Mortalità è definito come rapporto tra numero di decessi e dimensione della popolazione esposta.

Al 24 Aprile, la Protezione Civile dichiara 25.549 Decessi e 189.973 Casi Totali [1]. II Tasso di Letalità grezzo, pertanto, vale circa 0,13 (25.549/189.973) ovvero, equivalentemente, $13 \%$.

II suddetto valore è manifestamente superiore a quello valutato per gli altri paesi della Comunità Europea: al 10 aprile, il Tasso di Letalità grezzo valeva circa 9\% in Spagna, $8,6 \%$ in Olanda, $8 \%$ nel Regno Unito, $7,1 \%$ in Francia, $1,2 \%$ in Germania [2]. Tanto per l'Italia quanto per il resto dei paesi della Comunità Europea, II Tasso di Letalità grezzo restituisce un valore apparente, siccome calcolato ignorando decessi e contagi occulti (quest'ultimi da ricondursi a individui asintomatici ovvero paucisintomatici non sottoposti a tampone).

\subsection{Coefficienti di Correzione e Tasso di Letalità Effettivo}

Effettuiamo una stima del Tasso di Letalità effettivo per 'I'Italia.

I decessi dichiarati sono inferiori a quelli reali. Questa condizione è, di fatto innegabile. Molti decessi, infatti, sono stati occultati, in specie durante le fasi iniziale dell'epidemia. Avvalendosi dei dati ISTAT e Protezione Civile, il Coefficiente di Correzione dei Decessi stimato è pari a 2,65 [3].

Anche il numero dei contagiati, come platealmente dichiarato dallo stesso Borrelli nel corso d'una delle conferenze stampa della Protezione Civile, è da rivedersi al rialzo. Da tempo, disponiamo di stime che stanno rivelandosi sempre più attendibili. Faremo riferimento al Report n.13 dell'Imperial College (periodo d'ispezione: 25 Febbraio - 28 Marzo) [4].

Al 28 Marzo, il 9.8\% della popolazione italiana (circa 60 Milioni d'individui) avrebbe già contratto il virus. In altre parole, gli individui contagiati, al 28 Marzo, sarebbero stati 5.880.00. Tutte le stime stanno grossomodo convergendo verso questo valore: è agevole verificare come, al 30 Marzo, il numero dei contagi stimati vada dai 5 ai 6 Milioni di persone [5].

Per stimare il Coefficiente di Correzione dei Contagi, andiamo ai dati relativi al 28 Marzo.

È agevole realizzare come il numero di contagi dichiarati, al 28 Marzo, sia pari a 92.472 [1].

Pertanto, il Coefficiente di Correzione dei Contagi è pari a circa 63,6 (5.880.000/92.472): ciò significa che, per ottenere la stima dei contagi effettivi, è necessario moltiplicare il numero dei contagi dichiarati per 63,6. Tale numero, a primo acchito, potrebbe apparire eccessivo: a tal proposito, si sottolinea come, a detta d'un gruppo di ricerca dell'Università Statale di Milano diretto dal prof. La Vecchia, il numero dei contagi reali potrebbe risultare fino a 100 volte superiore rispetto a quello dei contagi dichiarati [5]. 
$\|$ Coefficiente di Correzione Totale è pari a circa 0,04 . Tale valore è banalmente ottenuto dividendo il Coefficiente di Correzione dei Decessi per quello dei Contagi. Possiamo pertanto affermare come il Tasso di Letalità Reale sia pari al $4 \%$ di quello deducibile dai dati forniti dalla Protezione Civile. Esplicitamente, il Tasso di Letalità reale varrebbe $0,005(0,13 \times 0,04)$ ovvero, equivalentemente, $0,5 \%$.

Al momento, la letalità del COVID-19 resta maggiore rispetto a quella dell'influenza stagionale, sebbene manifestamente dello stesso ordine di grandezza.

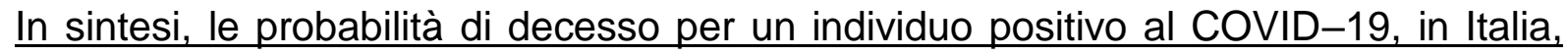
sono circa 5 su 1000 .

In seguito, vedremo come tale valore sia destinato a ridursi sensibilmente, a valle di un'opportuna caratterizzazione dei pazienti deceduti.

\section{Alcuni Termini di Paragone}

\subsection{Tabagismo}

Per meglio comprendere il reale significato del dato appena ricavato, stimeremo anzitutto il Tasso di Letalità del tabagismo.

In Italia, circa 1 individuo su 4 è fumatore [22\% - 25\%] [6,7]: pertanto, su una popolazione di 60 Milioni di abitanti, si contano dai 13.200 .000 ai 15.000 .000 di tabagisti.

Sempre in Italia, il fumo miete, ogni anno, dalle 70.000 alle 83.000 vittime. L'incidenza del fumo passivo verrà in questa sede ritenuta trascurabile, è ciò in virtù delle numerose limitazioni cui, a partire dal 2003 [8], i fumatori sono stati assoggettati: a tal proposito, oltre alle ben note limitazioni che interessano finanche alcuni spazi aperti, il divieto tassativo di fumare è stato recentemente esteso anche ai conducenti di autoveicoli, nonché ai passeggeri a bordo degli stessi, qualora ci si trovi in presenza di minorenni e donne in stato interessante.

II Tasso di Letalità, in questo caso, è offerto dal rapporto tra numero di decessi, a causa del fumo, e numero di fumatori. II valore del suddetto è compreso tra 0,005 $(70.000 / 15.000 .000)$ e $0,006(83.000 / 13.200 .000)$ ovvero, equivalentemente, tra $0,5 \%$ e $0,6 \%$.

In sintesi, le probabilità di decesso per un fumatore, in Italia, vanno dalle 5 alle 6 su 1000 , potendo pertanto risultare già maggiori di quelle precedentemente valutate per ipositivi COVID-19.

II fumo, assai evidentemente, costituisce un rischio addirittura superiore rispetto al COVID-19: tuttavia, com'è noto, nessun provvedimento indirizzato al divieto assoluto della vendita del tabacco è mai stato anche lontanamente contemplato.

A ulteriore legittimazione delle severissime restrizioni cui la popolazione resta, ancora oggi, di fatto assoggettata, si è fatto spesso ricorso al numero di vite risparmiate: a quanto stimato dall'Imperial College [4], il cosiddetto lockdown avrebbe consentito di salvare 38.000 vite.

L'importanza dei 38.000 decessi evitati è fuori discussione, questo sia chiaro, e vale ampiamente gli sforzi compiuti dalla popolazione negli ultimi due mesi. Tuttavia, altrettanto innegabile è l'importanza delle 83.000 vite che, ogni anno, potrebbero 
essere agevolmente salvate avversando opportunamente il tabagismo. Basterebbe, ancora una volta, vietare tassativamente la vendita del tabacco. Di certo, un intero settore, al lordo dell'indotto, verrebbe irreversibilmente polverizzato: tuttavia, si stima l'emergenza COVID-19 abbia ridotto in povertà assolute circa 10 Milioni d'individui [9], annientando, forse irreversibilmente, settori cruciali per l'economia italiana, quali turismo, ristorazione, spettacolo.

Qualcuno, tra gli ex fumatori, ingrasserebbe in virtù delle inevitabili modificazioni metaboliche correlate all'astinenza da tabacco: tuttavia, pare una parte della popolazione stia mettendo peso anche adesso, per via dell'impossibilità di dedicarsi alle ordinarie attività lavorative ovvero ricreative.

Qualcuno, tra gli ex fumatori, necessiterebbe di supporto psicologico al fine d'affrontare le conseguenze dell'astinenza da tabacco: tuttavia, al di là di ogni ragionevole dubbio, pare ottima parte della popolazione necessiti dello stesso tipo di supporto anche ora, viste le drammatiche conseguenze dell'isolamento forzato. Ma mai, al fine di salvare quelle 83.000 vite, gli italiani patirebbero ciò che stanno patendo ora per averne salvate, come era giusto fosse, 38.000 .

\subsection{Incidenti Stradali}

Per disporre d'un ulteriore termine di paragone, stimiamo il Tasso di Letalità per gli incidenti stradali [10].

Nel 2014, in Italia si sono verificati 177.031 sinistri con lesioni a persone, cui è conseguito il decesso di 3.381 individui (limitandosi ai casi accertati entro il trentesimo giorno) $[10,11]$.

La Pseudo Letalità è offerta dal rapporto tra il numero dei decessi e quello dei sinistri: pertanto, otteniamo il valore approssimato 0,02 (3.381/177.031).

Per ottenere un Tasso di Letalità propriamente inteso, è necessario esprimere i sinistri in termini d'individui coinvolti. Ipotizzando una media di 4 individui per sinistro, otteniamo un Tasso di Letalità pari a 0,005 (0,02/4) ovvero, equivalentemente, $0,5 \%$. In sintesi, le probabilità di decesso per un individuo coinvolto in un sinistro stradale, in Italia, sono pari a circa 5 su 1000, risultando esattamente identiche a quelle precedentemente valutate per i positivi al COVID-19.

\subsection{Incidenti Domestici}

Per completezza, stimiamo qualitativamente il Tasso di Letalità per gli incidenti domestici in Italia.

Nel 2008, i morti stimati per incidente domestico in Italia sono stati 5.783 [12]. Agni anno, gli accessi al pronto soccorso certificati a causa di incidente domestico sono circa 1.700.000 [12].

$\Perp$ Tasso di Letalità è grossomodo pari a 0,003 (5.783/1.700.000) ovvero, equivalentemente, $0,3 \%$. II valore è da ritenersi sottostimato, in quanto il fenomeno sembra essere in considerevole aumento, in specie tra donne e anziani [13].

In sintesi, le probabilità di decesso per un individuo coinvolto in un incidente domestico, in Italia, sono superiori a 3 su 1000, lievemente minori rispetto a quelle valutate per i positivi al COVID-19, sebbene dello stesso ordine di grandezza. 
Si riporta la sottostante tabella riassuntiva, comprensiva delle probabilità di decesso per altre patologie.

\begin{tabular}{c|c} 
Patologia o Condizione di Partenza & Probabilità di Decesso \\
\hline Epatite B [14] & 1 su 100 \\
Meningite Meningococcica [15] & 13 su 100 \\
Cancro (trattato) [16] & 30 su 100 \\
Influenza Spagnola (1918 - trattata) [17] & oltre 25 su 1.000 \\
Tabagismo & da 5 a 6 su 1.000 \\
COVID-19 & 5 su 1.000 \\
Incidente Stradale & 5 su 1.000 \\
Incidente Domestico & 3 su 1.000
\end{tabular}

\section{Caratterizzazione dei Pazienti Deceduti}

\subsection{Caratterizzazione per Comorbilità}

I pazienti deceduti presentano, in media, 3,3 patologie croniche preesistenti (diagnosticate prima di contrarre l'infezione da SARS-CoV-2) [18]. La comorbilità è valutata considerando, in via esclusiva, patologie di particolare rilievo, quali ipertensione arteriosa, diabete, insufficienza renale cronica, manifesta obesità. A titolo d'esempio, un paziente sofferente di reflusso gastroesofageo ovvero di micosi è da considerarsi sano. Solo il 3,6\% dei pazienti deceduti presenta 0 patologie preesistenti. Pertanto, ragionando in termini frequentistici, la probabilità che un paziente deceduto a causa del COVID-19 fosse inizialmente sano è pari a 0,036 (36 su 1000).

\subsection{Caratterizzazione per Età}

L'età media dei pazienti deceduti è pari a 79 anni [18]. I decessi si concentrano notevolmente nella fascia più anziana della popolazione [18]. Nell' $1 \%$ dei casi, i pazienti deceduti hanno tra i 40 e i 49 anni [18]. Nel $4 \%$ dei casi, i pazienti deceduti hanno tra i 50 e i 59 anni [18]. Sommando le due categorie precedenti, è possibile affermare come nel $5 \%$ dei casi i pazienti deceduti abbiano un'età compresa tra i $40 \mathrm{e}$ i 59 anni.

I pazienti deceduti con età inferiore ai 40 anni sono in percentuale ampiamente trascurabile [18].

Approssimativamente, pertanto, è possibile affermare come nel $5 \%$ dei casi i pazienti deceduti abbiano meno di 60 anni. Tale categoria verrà in questa sede considerata al solo fine d'offrire uno scenario verosimile: tra le varie ipotesi ancora contemplate per la cosiddetta "fase 2", infatti, è da annoverarsi la possibilità di garantire temporanea prosecuzione alle restrizioni per i soli cittadini ultrasessantenni, siccome ritenuti maggiormente esposti alle conseguenze dell'infezione.

Ragionando in termini frequentistici, la probabilità che un paziente deceduto a causa del COVID-19 abbia un'età inferiore ai 60 anni è pari a 0,05 (5 su 100). 


\subsection{Caratteristiche Combinate}

Combiniamo i dati finora ottenuti, moltiplicando tra di loro i valori di probabilità.

A valle dell'infezione, la probabilità di decesso per un individuo inizialmente sano è pari a circa 0,00018 .

II risultato è stato ottenuto moltiplicando il Tasso di Letalità reale $(0,005)$ per la probabilità che un paziente deceduto a causa del COVID-19 fosse inizialmente sano $(0,036)$.

In definitiva, un individuo sano, una volta infettatosi, ha 18 probabilità su 10.000 di morire.

A valle dell'infezione, la probabilità di decesso per un individuo di età inferiore ai 60 anni è pari a 0,00025 .

II risultato è stato ottenuto moltiplicando il Tasso di Letalità reale $(0,005)$ per la probabilità che un paziente deceduto a causa del COVID-19 abbia un'età inferiore ai 60 anni $(0,05)$.

In altre parole, un individuo d'età inferiore a 60 anni, una volta infettatosi, ha 25 probabilità su 10.000 di morire.

A valle dell'infezione, la probabilità di decesso per un individuo sano d'età inferiore ai 60 anni è pari a circa 0,000009 . II risultato è stato ottenuto moltiplicando, tra di loro, il Tasso di Letalità reale $(0,005)$, la probabilità che un paziente deceduto a causa del COVID-19 fosse inizialmente sano $(0,0036)$ e la probabilità che un paziente deceduto a causa del COVID-19 abbia un'età inferiore ai 60 anni $(0,05)$.

In altre parole, un individuo sano d'età inferiore a 60 anni, una volta infettatosi, ha 9 probabilità su 100.000 di morire. II valore è da ritenersi approssimato siccome la distribuzione delle comorbilità non è uniforme: in altri termini, il numero di patologie preesistenti cresce, anche sensibilmente, con l'età del paziente [18].

Parliamo di probabilità condizionate, assai evidentemente. La condizione di partenza è che il paziente abbia già contratto il virus. Proviamo a rimuovere, assai qualitativamente, tale condizione.

Supponiamo un individuo NON infetto venga a contatto con un infetto. II contagio non può essere né escluso né dato per certo in maniera categorica. Pertanto, riterremo la probabilità d'infettarsi sia sempre maggiore di 0 e minore di 1 . La probabilità che il primo individuo vanga infettato dal secondo dipende, tra le varie, da durata e modalità del contatto: in altre parole, fattori cruciali sono costituiti dal tempo di permanenza in prossimità dell'infetto, nonché dal valore della distanza interpersonale [19].

Quale intervallo di probabilità, in assenza di ulteriori informazioni, consideriamo 0,10,9 . Se la positività non è data per scontata, le probabilità di decesso vanno moltiplicate per i suddetti valori.

Pertanto, un individuo sano di età inferiore ai 60 anni, NON ancora infetto, ha da 9 a 80 probabilità su 1.000 .000 di contrarre il virus e morire, in seguito, a causa di esso. 
II valore di probabilità è ottenuto moltiplicando gli estremi dell'intervallo $[0,1-0,9]$ per il valore dedotto in precedenza (9 probabilità su 100.000).

Si riporta la sottostante tabella, al fine di richiamare la totalità dei valori finora dedotti.

Italia (valori approssimati)

\begin{tabular}{|c|c|c|c|}
\hline $\begin{array}{l}\text { Condizione di } \\
\text { Partenza }\end{array}$ & $\begin{array}{c}\text { Condizione } \\
\text { Accessoria A }\end{array}$ & $\begin{array}{c}\text { Condizione } \\
\text { Accessoria B }\end{array}$ & $\begin{array}{l}\text { Probabilità di } \\
\text { Decesso }\end{array}$ \\
\hline $\begin{array}{c}\text { Positivo } \\
\text { al COVID-19 }\end{array}$ & l & I & 5 su 1.000 \\
\hline $\begin{array}{l}\text { Positivo } \\
\text { al COVID-19 }\end{array}$ & $\begin{array}{l}\text { Età Inferiore } \\
\text { ai } 60 \text { Anni }\end{array}$ & l & 25 su 10.000 \\
\hline $\begin{array}{l}\text { Positivo } \\
\text { al COVID-19 }\end{array}$ & / & $\begin{array}{l}\text { Sano PRIMA } \\
\text { del Contagio }\end{array}$ & 18 su 10.000 \\
\hline $\begin{array}{l}\text { Positivo } \\
\text { al COVID-19 }\end{array}$ & $\begin{array}{l}\text { Età Inferiore } \\
\text { ai } 60 \text { Anni }\end{array}$ & $\begin{array}{l}\text { Sano PRIMA } \\
\text { del Contagio }\end{array}$ & 9 su 100.000 \\
\hline $\begin{array}{l}\text { NON Positivo } \\
\text { al COVID-19 }\end{array}$ & $\begin{array}{c}\text { Età Inferiore } \\
\text { ai } 60 \text { Anni }\end{array}$ & $\begin{array}{l}\text { Sano PRIMA } \\
\text { del Contagio }\end{array}$ & $\begin{array}{c}\text { da } 9 \text { a } 80 \text { su } \\
1 \text { Milione }\end{array}$ \\
\hline
\end{tabular}

I suddetti valori sono ulteriormente assoggettabili a riduzione anche sensibile.

Infatti, il Tasso di Letalità grezzo, dal quale si è partiti al fine d'ottenere i risultati sintetizzati nella tabella precedente, varia notevolmente da regione a regione, da provincia a provincia.

Riportiamo sinteticamente i risultati per la Campania, per la quale il Tasso di Letalità grezzo è pari a $0,08[20]$.

Regione Campania (valori approssimati)

\begin{tabular}{|c|c|c|c|}
\hline $\begin{array}{l}\text { Condizione di } \\
\text { Partenza }\end{array}$ & $\begin{array}{c}\text { Condizione } \\
\text { Accessoria A }\end{array}$ & $\begin{array}{c}\text { Condizione } \\
\text { Accessoria B }\end{array}$ & $\begin{array}{l}\text { Probabilità di } \\
\text { Decesso }\end{array}$ \\
\hline $\begin{array}{l}\text { Positivo } \\
\text { al COVID-19 }\end{array}$ & / & I & 3 su 1.000 \\
\hline $\begin{array}{l}\text { Positivo } \\
\text { al COVID-19 }\end{array}$ & $\begin{array}{l}\text { Età Inferiore } \\
\text { ai } 60 \text { Anni }\end{array}$ & I & 15 su 10.000 \\
\hline $\begin{array}{l}\text { Positivo } \\
\text { al COVID-19 }\end{array}$ & I & $\begin{array}{l}\text { Sano PRIMA } \\
\text { del Contagio }\end{array}$ & 11 su 10.000 \\
\hline $\begin{array}{l}\text { Positivo } \\
\text { al COVID-19 }\end{array}$ & $\begin{array}{l}\text { Età Inferiore } \\
\text { ai } 60 \text { Anni }\end{array}$ & $\begin{array}{l}\text { Sano PRIMA } \\
\text { del Contagio }\end{array}$ & 6 su 100.000 \\
\hline $\begin{array}{l}\text { NON Positivo } \\
\text { al COVID-19 }\end{array}$ & $\begin{array}{c}\text { Età Inferiore ai } 60 \\
\text { Anni }\end{array}$ & $\begin{array}{c}\text { Sano PRIMA del } \\
\text { Contagio }\end{array}$ & $\begin{array}{c}\text { da } 6 \text { a } 50 \mathrm{su} \\
1 \text { Milione }\end{array}$ \\
\hline
\end{tabular}




\section{L’Anomalia Lombarda}

Ancora oggi, la Lombardia rappresenta senza dubbio una singolarità degna di nota. Per comprenderlo, basti pensare al triste primato dei connazionali lombardi: circa il $50 \%$ dei decessi sul totale [20].

Riportiamo sinteticamente i risultati per la Lombardia, per la quale il Tasso di Letalità grezzo è pari a $0,18[20]$.

Regione Lombardia (valori approssimati)

\begin{tabular}{c|c|c|c}
\hline $\begin{array}{c}\text { Condizione di } \\
\text { Partenza }\end{array}$ & $\begin{array}{c}\text { Condizione } \\
\text { Accessoria A }\end{array}$ & $\begin{array}{c}\text { Condizione } \\
\text { Accessoria B }\end{array}$ & $\begin{array}{c}\text { Probabilità di } \\
\text { Decesso }\end{array}$ \\
\hline $\begin{array}{c}\text { Positivo } \\
\text { al COVID-19 }\end{array}$ & $/$ & $/$ & 7 su 1.000 \\
$\begin{array}{c}\text { Positivo } \\
\text { al COVID-19 }\end{array}$ & $\begin{array}{c}\text { Età Inferiore } \\
\text { ai 60 Anni }\end{array}$ & $/$ & 35 su 10.000 \\
Positivo & $/$ & $\begin{array}{c}\text { Sano PRIMA } \\
\text { del Contagio }\end{array}$ & 25 su 10.000 \\
COVID-19 & Età Inferiore & $\begin{array}{c}\text { Sano PRIMA } \\
\text { del Contagio }\end{array}$ & 13 su 100.000 \\
al COVID-19 & $\begin{array}{c}\text { ai 60 Anni } \\
\text { NON Pòn Inferiore } \\
\text { al COVID-19 }\end{array}$ & $\begin{array}{c}\text { Sano PRIMA } \\
\text { del Contagio }\end{array}$ & $\begin{array}{c}\text { da } 13 \text { a } 117 \text { su } \\
1 \text { Milione }\end{array}$ \\
\hline
\end{tabular}

Identificare un'intera nazione con la più sfortunata tra le sue regioni potrebbe portare a risultati oltremodo fuorvianti.

Infatti, l'elevatissimo numero di decessi in Lombardia (13.575) [20], in specie alla luce di quanto finora dedotto, non sarebbe da ricondursi alla letalità del COVID-19, bensì ai seguenti fattori:

a. La presenza del virus è stata rilevata assai tardivamente. Pare ormai certo come il SARS-CoV -2 abbia raggiunto I'Italia nel mese di Gennaio, se non addirittura in precedenza [21].

La Lombardia ha inconsapevolmente convissuto con il virus, in tal modo agevolandone notevolmente la diffusione.

Per troppo tempo, i cittadini hanno serenamente garantito prosecuzione alla propria ordinaria quotidianità: totale libertà di spostamento (trasporti pubblici in piena operatività) e di assembramento (dai bar ai ristoranti, dalle discoteche agli stadi), fabbriche a pieno regime, operatori sanitari, tra i primi vettori del contagio, non allertati (medici e infermieri, cui non è stata di fatto imposta l'adozione d'alcun protocollo d'emergenza, hanno continuato a svolgere le proprie mansioni privi d'opportuni dispositivi di protezione individuale). 
b. I medici non possedevano, inizialmente, conoscenze sufficienti a trattare la patologia in modo adeguato. II "mantra" della polmonite interstiziale bilaterale, ostinatamente recitato dai medici in prima linea, è progressivamente decaduto, cedendo il posto a quello che può essere definito un "danno multiorgano" derivante, prevalentemente, da coagulopatia intravasale diffusa e ipercitochinemia [22]. Attualmente, disponiamo di armi numerose ed efficaci per trattare, al netto di rare eccezioni, una vasta gamma di pazienti affetti da COVID-19, dall'eparina a basso peso molecolare [23] al tocilizumab [24], tanto per citarne un paio. Allo scoppiare dell'epidemia, ci si è giocoforza limitati a sottoporre i pazienti più critici a ventilazione meccanica invasiva: in alcuni casi, tuttavia, tale pratica è equivalsa a soffiare aria in un vaso di terracotta (sperando potesse gonfiarsi), ottenendo, nel migliore dei casi, praticamente nulla.

c. Una volta esplosa l'emergenza, i nosocomi, già sovraffollati in virtù delle gravissime condizioni dei primi pazienti ricoverati, sono stati presi d'assalto dai cittadini, terrorizzati dal repentino cambiamento di scenario. Occorre ricordare come la popolazione abbia inizialmente trovato conforto, ritenendosi imprudentemente fuori pericolo, nelle dichiarazioni rassicuranti di esperti quali Roberto Burioni ("In Italia il rischio è zero: il virus non circola") [25] e Massimo Galli ("In Italia la situazione è sotto controllo [...] la malattia da noi difficilmente potrà diffondersi') [26]. Persino L'Organizzazione Mondiale della Sanità, nel Report n. 46 del 6 Marzo, minimizzava dichiarando: "Dal punto di vista della salute pubblica, le misure da implementare per limitarne la diffusione (il riferimento è al COVID-19) sono le stesse (il riferimento è all'influenza stagionale): igiene delle mani, starnutire all'interno del gomito o in un fazzoletto di carta e poi gettarlo" [27]. lo shock, pertanto, è stato davvero notevole. La corsa ai nosocomi ha sensibilmente contribuito alla diffusione del contagio, nonché al sovraccarico d'un Sistema Sanitario del tutto impreparato a fronteggiare una simile emergenza. Considerata la portata del fenomeno, la sanità lombarda, fortunatamente tra le migliori in Italia, non avrebbe potuto reagire meglio.

d. II Servizio Sanitario Nazionale, nonostante alcune relative eccellenze, era da ritenersi claudicante ben prima dell'esplosione dell'emergenza. In Italia, nel 2017, risultavano disponibili, per degenza ordinaria, 151.646 posti letto in ospedali pubblici (2,5 ogni 1.000 abitanti) e 40.458 in strutture private $(0,7$ ogni 1.000 abitanti). Complessivamente, pertanto, 3,2 posti letto ogni 1.000 abitanti [28]. Nel 2018, in Italia, sommando pubblico e privato, i posti letto complessivi destinati alla terapia intensiva ammontavano a 5.090: in pratica, circa 8,42 ogni 100.000 abitanti [28]. Già nel 2017, quello Italiano rappresentava il sesto dato più basso dell'Unione Europea, caratterizzata da una media di 5 posti letto per degenza ordinaria ogni 1.000 abitanti [28]. 
e. La Lombardia è tra le regioni più inquinate del paese. A tal proposito, Ernesto Burgio afferma: " $A$ causa dell'inquinamento, gli adulti e in particolare gli anziani che soffrono di malattie croniche sono affetti da infiammazione persistente di basso grado e disfunzione endoteliale sistemica, che sembra essere il più potente fattore predisponente e scatenante per la tempesta di citochine e la disfunzione trombotica" [29].

\section{Considerazioni Finali}

Come riferito dall'AIFA, "il decorso clinico del COVID-19 sta sempre più nettamente delineando l'esistenza di tre distinte fasi cliniche della malattia. [...] (La prima fase) si caratterizza clinicamente per la presenza di malessere generale, febbre e tosse secca. I casi in cui si riesce a bloccare l'infezione in questo stadio hanno un decorso assolutamente benigno" [23]. Oramai, non è ragionevole ricondurre la sintomatologia di cui sopra all'influenza stagionale, per la quale il periodo d'ispezione raramente lambisce la diciassettesima settimana [30]. Pertanto, sarebbe auspicabile provvedere alla celere individuazione dei pazienti sintomatici, al fine d'arrestare il decorso della malattia agli esordi: adesso, abbiamo gli strumenti per farlo.

II Tasso di Letalità reale del COVID-19 valutato in questa sede, appare già di per sé addirittura maggiore di quello del tabagismo, nonché identico a quello stimato per gl'incidenti stradali. E ciò pure al netto di qualsivoglia caratterizzazione dei pazienti positivi, a valle della quale la riduzione del suddetto tasso è plateale. Inoltre, il Coefficiente di Correzione dei Decessi $(2,65)$ potrebbe essere stato sovrastimato, in quanto, con l'evolversi dell'epidemia, i decessi occulti sono da considerarsi naturalmente diminuiti. Al contrario, il Coefficiente di Correzione dei Contagi $(63,6)$ potrebbe essere stato oggetto di sottostima, essendo da alcuni ritenuto addirittura prossimo a 100 [5]. Ne consegue una verosimile sottostima del Coefficiente di Correzione Totale $(0,04)$, cui deriva una sovrastima del Tasso di Letalità reale. In altre parole, il Tasso di Letalità del COVID-19 potrebbe rivelarsi addirittura inferiore rispetto a quello in questa sede dedotto $(0,005)$.

In virtù di quanto osservato, ci si chiede se valga o meno concretamente la pena continuare a sottoporre il paese a restrizioni (tra l'altro indiscriminate, a dispetto della marcata variabilità degli scenari sul territorio) la cui severità, di fatto, sta irreversibilmente polverizzando interi settori economici, riducendo in povertà milioni d'italiani. Ammesso sia davvero utile, in termini epidemiologici, limitare in maniera estrema la diffusione del contagio [31,32], ci si chiede per quale motivo l'adozione di metodologie efficaci di screening e prevenzione, pure abbinate a una parziale modificazione, meno castigante, dei nostri comportamenti abituali, non possa costituire una valida alternativa al prolungamento delle attuali condizioni restrittive. Tanto più siccome, al di là di ogni ragionevole dubbio, i tempi necessari all'ottenimento d'un vaccino, al netto di considerazioni inerenti efficacia del prodotto e rischi correlati alla sua somministrazione [33], sono tutto fuorché compatibili con una strategia prevalentemente attendista. 


\section{Riferimenti}

1. Banca Dati in Aggiornamento Costante. Coronavirus in Italia: i dati e la mappa. II Sole 24 Ore - Lab24.

https://lab24.ilsole24ore.com/coronavirus/

2. Greco, P. (2020) Perché in Germania si muore di meno? II Bo Live - Università di Padova - Scienza e Ricerca.

https://ilbolive.unipd.it/it/news/perche-germania-si-muore-meno

3. Autori Vari (2020) Numero di Decessi per COVID19 - Possibile che siano quasi tre volte più numerosi dei dati ufficiali. VISION - C. Stampa del 2 Aprile 2020. https://www.thinktank.vision/images/Stima decessi COVID19 020420.pdf

4. Flaxman, S., Mishra, S., Gandy, A. et al. (2020) Report 13 - Estimating the number of infections and the impact of non-pharmaceutical interventions on COVID-19 in 11 European countries. Imperial College - London.

https://www.imperial.ac.uk/mrc-global-infectious-disease-analysis/covid-

19/report-13-europe-npi-impact/

5. De Giorgio, T. (2020) La ricerca dell'Università Statale: "I contagi reali in Italia? Potrebbero essere 5 milioni". La Repubblica, 3 Aprile 2020.

https://rep.repubblica.it/pwa/locali/2020/04/03/news/milano la ricerca dell uni versita statale i contagi reali in italia potrebbero essere 5 milioni 253027121/

6. Ministero della Salute (2014) Prevenzione e Controllo del Tabagismo Distribuzione percentuale del campione in base all'abitudine al fumo. OSSFAD - Indagine DOXA-ISS 2014.

http://www.salute.gov.it/imgs/C 17 pubblicazioni 2173 allegato.pdf

7. Ministero della Salute (2017) Prevenzione e Controllo del Tabagismo Rapporto 2017. Direzione Generale Della Prevenzione Sanitaria - Ufficio 8. http://www.salute.gov.it/imgs/C 17 pubblicazioni 2853 allegato.pdf

8. Legge 16 gennaio 2003, n. 3, Capo IX, Disposizioni in Materia di Tutela della Salute, art. 51

https://www.camera.it/parlam/leggi/03003I.htm

9. Raimo, A. (2020) Coronavirus, Centro studi Ugl: "Rischio quasi 10 milioni in povertà assoluta". DiRE - Agenzia Stampa Nazionale, 26 Aprile 2020.

https://www.dire.it/26-04-2020/452361-coronavirus-centro-studi-ugl-rischioquasi-10-milioni-in-poverta-assoluta/

10. Incidenti Stradali - Aspetti epidemiologici. EpiCentro - II portale dell'epidemiologia per la sanità pubblica, a cura dell'Istituto Superiore di Sanità. https://www.epicentro.iss.it/stradale/epidemiologia

11. Rapporti ISTAT - ACI. http://www.aci.it/laci/studi-e-ricerche/dati-e-statistiche/incidentalita.html

12. Ministero della Salute (2011) La situazione sanitaria del Paese: Incidenti Domestici.

http://www.rssp.salute.gov.it/rssp/paginaParagrafoRssp.jsp?sezione=situazion e\&capitolo $=$ mortalita\&id $=2678$ 
13. Gli incidenti domestici: le statistiche ISTAT sottolineano un fenomeno in crescita. Globalist, 10 Giugno 2019.

https://www.globalist.it/news/2019/06/10/gli-incidenti-domestici-le-statisticheistat-sottolineano-un-fenomeno-in-crescita-2042663.html

14. Epatite Virale - Epatite B. EpiCentro - II portale dell'epidemiologia per la sanità pubblica, a cura dell'Istituto Superiore di Sanità.

https://www.epicentro.iss.it/epatite/epatite-b

15. Ciofi degli Atti M., Salmaso, S. Meningiti da Meningococco in Italia. EpiCentro - II portale dell'epidemiologia per la sanità pubblica, a cura dell'ISS.

https://www.epicentro.iss.it/meningite/Meningiti\%20da\%20meningococco\%20i n\%20ltalia.pdf

16. Shimkin, M. B., Griswold, M. H., Cutler, S. J. (1984) Survival in untreated and treated cancer. CA: a cancer journal for clinicians, 34(5), 282-294.

https://doi.org/10.3322/canjclin.34.5.282

17. La sorveglianza PASSI - Le Pandemie Influenzali del Ventesimo Secolo. EpiCentro - Il portale dell'epidemiologia per la sanità pubblica, a cura dell'Istituto Superiore di Sanità.

https://www.epicentro.iss.it/passi/storiePandemia

18. Report sulle caratteristiche dei pazienti deceduti positivi all'infezione da SARSCoV-2 in Italia (aggiornamento del 23 Aprile 2020). EpiCentro - II portale dell'epidemiologia per la sanità pubblica, a cura dell'Istituto Superiore di Sanità. https://www.epicentro.iss.it/coronavirus/sars-cov-2-decessi-italia

19. Roddel, S. (2020) COVID-19: Econometrics researcher's calculations illustrate why we should social distance. PreventionWeb - University of Notre Dame. https://www.preventionweb.net/news/view/71160

20. Ministero della Salute. Nuovo Coronavirus. COVID-19. Aggiornato al 24 Aprile 2020, ore 17:00.

http://www.salute.gov.it/imgs/C 17 notizie 46320 file.pdf

21. Bucci, E., Marinari, E. (2020) L'evoluzione dell'epidemia da coronavirus in Italia. Scienza in rete.

https://www.scienzainrete.it/articolo//\%E2\%80\%99evoluzionedell\%E2\%80\%99epidemia-da-coronavirus-italia/enrico-bucci-enzomarinari/2020-03-02

22. COVID19: l'esito dell'infezione si decide nei primi 10-15 giorni dal contagio. Istituto Superiore di Sanità, Ufficio Stampa - Primo Piano. Discussione del PrePrint "The First, Comprehensive Immunological Model of COVID-19: Implications for Prevention, Diagnosis, and Public Health Measures" (Matricardi, P., Dal Negro, R., Nisini, R.), approvato in data 24 Aprile 2020. https://www.preprints.org/manuscript/202004.0436/v1

23. Scheda informativa AIFA. Eparine a basso peso molecolare nei pazienti adulti con COVID-19.

https://www.aifa.gov.it/documents/20142/1123276/Eparine Basso Peso Mole colare 11.04.2020.pdf/e30686fb-3f5e-32c9-7c5c-951cc40872f7 
24. Fascicoli AIFA sulle sperimentazioni autorizzate. Tocilizumab in patients with severe COVID-19 pneumonia. Sezione "Documenti relativi alle sperimentazioni cliniche in corso". https://www.aifa.gov.it/sperimentazioni-cliniche-covid-19

25. adnKronos. Virus, Burioni: "In Italia il rischio è zero". Nota del 2 Febbraio 2020. https://www.adnkronos.com/salute/2020/02/02/virus-burioni-italia-rischiozero 4dqZ5UUAKtxSKUIhRYlvqJ.html?refresh ce

26. Bosco, F. (2020) Coronavirus, Galli (Sacco): «In Italia situazione sotto controllo, misure assunte stanno contenendo fenomeno». Sanità Informazione, 10 Febbraio 2020.

https://www.sanitainformazione.it/salute/coronavirus-galli-direttore-malattieinfettive-sacco-primi-indicatori-positivi-da-due-giorni-in-cina-cresce-meno-initalia-soli-tre-casi-importati/

27. Landucci, M. (2020) Coronavirus. Che differenza c'è tra COVID-19 e una normale influenza? Le risposte dell'OMS. Quotidiano Sanità - Scienza e Farmaci, 7 Marzo 2020.

http://www.quotidianosanita.it/scienza-efarmaci/articolo.php?articolo id=82243

28. Q. F. (2020) Coronavirus, i medici delle terapie intensive in Lombardia: "Azioni tempestive o disastrosa calamità sanitaria". L'ipotesi delle priorità d'accesso: "Prima chi ha più probabilità di sopravvivenza". II Fatto Quotidiano, 7 Marzo 2020.

https://www.ilfattoquotidiano.it/2020/03/07/coronavirus-i-medici-delle-terapieintensive-in-lombardia-azioni-tempestive-o-disastrosa-calamita-sanitarialipotesi-delle-priorita-daccesso-prima-chi-ha-piu-probabilita-disopravvivenza/5729020/?fbclid=IwAR35ocB0MVCoL5TGtW95euy4VgM1Hmtl Cv4ZY1zNnA3TuR18CR-5Xv RQWcb

29. Burgio, E. (2020) COVID-19: il dramma italiano. Quattro fattori di rischio evitabili. Scienza e Tecnologia, 27 Aprile 2020.

https://wsimag.com/it/scienza-e-tecnologia/62062-covid-19-il-drammaitaliano?fbclid=IwAR0302GdXXyVqWQZcE0SZ8Wzops3DBzHf3ZrVipehOTWouB8FKGnOIYLd4

30. FluNews - Italia. Rapporto della sorveglianza integrata dell'influenza. EpiCentro - Il portale dell'epidemiologia per la sanità pubblica, a cura dell'ISS. https://www.epicentro.iss.it/influenza/flunews\#epi

31. Articolo non firmato (2020) La virologa Gismondo: "60-70\% di italiani sarà positivo, ma non preoccupiamoci". Huffpost-Culture, 13 Marzo 2020. https://www.huffingtonpost.it/entry/la-virologa-gismondo-60-70-di-italiani-sarapositivo-ma-non-preoccupiamoci it 5e6bba13c5b6bd8156f6b865

32. D'Amico, G. (2020) Covid-19, le domande del pubblico e della scienza. RaiNews24, Rubrica "Salute", 7 Marzo 2020 (in studio, l'epidemiologo D. Greco; in collegamento da Torino, E. Bucci - Temple University, USA). http://www.rainews.it/dl/rainews/media/Covid19-Le-domande-della-gentedella-scienza-a535688d-8dc1-48b8-b073-f1691461b5d8.html 
33. Camerieri, V. (2020) Ma i tamponi per verificare l'infezione funzionano? E come? \#Byoblu24: intervista alla prof.ssa Loretta Bolgan.

https://www.byoblu.com/2020/03/13/ma-i-tamponi-per-verificare-linfezionefunzionano-e-come-loretta-bolgan-byoblu24/

\section{About the Author:}

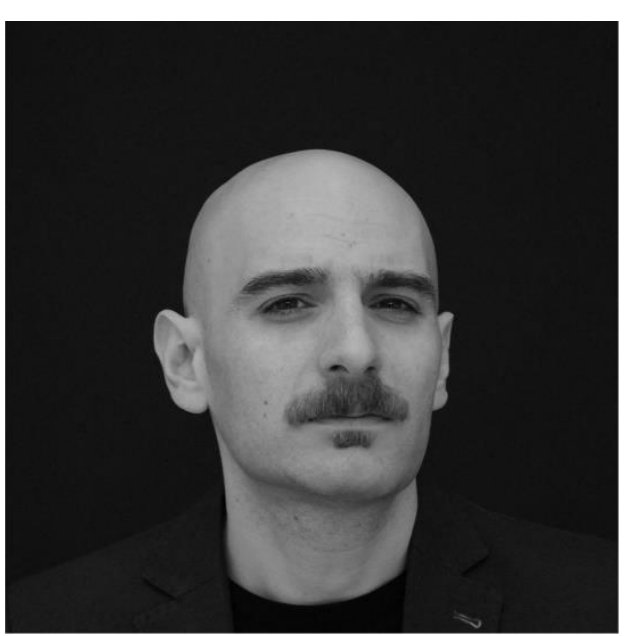

Carmine Cataldo was born on 13 February 1979 in Battipaglia (Salerno - Italy).

In 2004, he graduates from the University of Salerno in Mechanical Engineering; the same year, he passes the qualifying examination to the Engineers Register.

In 2008, he obtains, from the University of Salerno, a $\mathrm{PhD}$ in Mechanical Engineering, with a final dissertation based upon the analytical modelling of the extrusion and stretching processes targeted at thermoplastic polymers.

Carmine Cataldo is also a jazz pianist and composer. In 2018, he graduates (Master Degree, Summa Cum Laude) from the State Conservatory of Salerno in Disciplines of Jazz and Improvisation (Jazz Piano).

Carmine Cataldo is also a martial arts teacher. He is appointed "Shifu" by his own master, Sifu Antonello Parisi (ITKAA), on 18 December 2016. He is a Black Belt in Shaolin Wing Chun (Superior Instructor, 4th Technical Level) and Combat Escrima Concept (Advanced Instructor, 3rd Technical Level). 